\title{
Etude de la sensibilité aux antibiotiques des mycoplasmes isolés de spermes à Abidjan
}

\author{
Thérèse Brah KOUASSI-AGBESSI ${ }^{1,2^{*}}$, Asher Mian CABLAN ${ }^{1,3}$, \\ Anne Gouhonon KRIZO' ${ }^{1}$, Nanga Yesse ZINZENDORF ${ }^{1,3}$ et Christophe N'cho AMIN ${ }^{2,4}$ \\ ${ }^{1}$ Département de bactériologie-virologie, UFR Sciences Pharmaceutiques et Biologiques, Université Félix \\ Houphouët-Boigny, BPV 34 Abidjan, Côte d'Ivoire. \\ ${ }^{2}$ Institut National d'Hygiène Publique, BPV 14 Abidjan, Côte d'Ivoire. \\ ${ }^{3}$ Laboratoire National de la Santé Publique, 18 BP 2403 Abidjan 18, Côte d'Ivoire. \\ ${ }^{4}$ Département de Chimie Analytique, UFR Sciences Pharmaceutiques et Biologiques, Université Félix \\ Houphouët-Boigny, BPV 34 Abidjan, Côte d'Ivoire. \\ *Auteur correspondant ; E-mail : aktherese@hotmail.com; BPV 34 Abidjan, Côte d'Ivoire, \\ Tel : +22505952788
}

\section{RESUME}

Les mycoplasmes sont des bactéries du tractus uro-génital souvent associés à l'infertilité masculine. Au plan thérapeutique, des résistances acquises vis-à-vis des antibiotiques ont été rapportées. Ce travail avait pour objectif de déterminer le niveau de sensibilité des mycoplasmes uro-génitaux isolés au cours de la spermoculture. L'étude rétrospective, a porté sur 313 mycoplasmes identifiés grâce au kit Mycoplasma IST $_{2}$ (Biomérieux, Réf 42 505). Ce kit permet la quantification différentielle, l'identification de Ureaplasma urealyticum, et Mycoplasma hominis, ainsi que l'étude de la sensibilité à neuf antibiotiques (josamycine, azithromycine, érythromycine, clarithromycine, pristinamycine, tétracycline, doxycycline, ofloxacine etciprofloxacine). Ureaplasma urealyticum a été identifié dans 245 échantillons (78,3\%) et Mycoplasma hominis dans 27 (8,6\%). La co-infection (Ureaplasma urealyticum/Mycoplasma hominis) a été retrouvée chez 41 patients $(13,1 \%)$. La josamycine était active sur l'ensemble des germes avec des sensibilités respectives de 90,2\% et 92,6\% pour Ureaplasma urealyticum et pour Mycoplasma hominis. L'activité des cyclines sur Ureaplasma urealyticum était bonne tandis que celle des fluoroquinolones était médiocre. La pristinamycine était efficace sur Mycoplasma hominis (70\%). L'étude a montré que la josamycine était active sur l'ensemble des mycoplasmes, les cyclines conservaient une bonne efficacité sur Ureaplasma urealyticum contrairement aux fluoroquinolones. Ces variations de sensibilité justifient l'intérêt de l'identification des deux espèces et l'étude de la sensibilité pour une bonne prise en charge de ces infections.

(C) 2019 International Formulae Group. All rights reserved.

Mots clés : Mycoplasmes uro-génitaux, sperme, sensibilité, antibiotiques.

\section{Antibiotic susceptibility of urogenital mycoplasma strains isolated from sperms in Abidjan}

\begin{abstract}
Mycoplasma urogenital tract bacteria that are often associated with male infertility. At the therapeutic level, acquired resistance to antibiotics has been reported. The objective of this work was to determine the sensitivity level of urogenital mycoplasma strains isolated during sperm culture. Retrospective study focused
\end{abstract}


on 313 mycoplasma identified with Mycoplasma IST 2 kit (Biomérieux Ref 42 505). This kit allows differential quantification, identification of Ureaplasma urealyticum, and Mycoplasma hominis, as well as sensitivity studies to nine antibiotics (josamycin, azithromycin, erythromycin, clarithromycin, pristinamycin, tetracycline, doxycycline, ofloxacin and ciprofloxacin). Ureaplasma urealyticum was identified in 245 samples (78.3\%) and Mycoplasma hominis in 27 (8.6\%). Co-infection (Ureaplasma urealyticum/Mycoplasma hominis) was found in 41 patients (13.1\%). Josamycin was active on all germs with sensitivities of $90.2 \%$ and $92.6 \%$ respectively for Ureaplasma urealyticum and Mycoplasma hominis. Cycline activity on Ureaplasma urealyticum was good while fluoroquinolone activity was poor. Pristinamycin was effective on Mycoplasma hominis (70\%). The study showed that josamycin was active on all mycoplasma, cyclins retained good efficacy on Ureaplasma urealyticum unlike fluoroquinolones. These variations in sensitivity justify the interest of identifying the two species and studying the sensitivity for a good management of these infections.

(C) 2019 International Formulae Group. All rights reserved.

Keywords: Urogenital mycoplasma, sperm, suceptibility, antibiotics.

\section{INTRODUCTION}

L'infertilité masculine est un problème de santé bien connu dans les pays en développement (Nana, 2010). Les principales étiologies sont les infections génitales causées entre autre par les mycoplasmes (Abarikwu, 2013). Les mycoplasmes sont des bactéries appartenant à la flore commensale d'un très grand nombre d'individus et pouvant présenter un pouvoir pathogène occasionnel (Hartmann, 2009). Trois espèces sont fréquemment identifiées; il s'agit de Mycoplasma genitalium, Mycoplasma hominis et Ureaplasma urealyticum. Ces pathogènes occasionnels sont associés à diverses pathologies telles que les urétrites non gonococciques (Mycoplasma genitalium et Ureaplasma urealyticum), les cervicites et vaginoses bactériennes (Mycoplasma hominis), les infections néonatales (Ureaplasma urealyticum), les troubles de la reproduction (Mycoplasma hominis et Ureaplasma urealyticum) (Hartmann, 2009; Abdel Rahman et al., 2016). Selon plusieurs études, les mycoplasmes occupent une place non négligeable dans les infections urogénitales en Afrique sub-saharienne (Nwadiodaet al., 2010; Karou et al., 2012). $\mathrm{Au}$ plan thérapeutique, les antibiotiques potentiellement actifs préconisés dans le traitement des infections à mycoplasmes urogénitaux appartiennent aux familles des cyclines, des macrolides-lincosanides- synergistines et des fluoroquinolones (Waites et al., 2012). Par ailleurs, il a été rapporté l'apparition de résistance acquise vis-à-vis des antibiotiques couramment prescrits dans le traitement des infections à mycoplasmes (Waites et al., 2003 ; Farkas et al., 2011). Cependant, le niveau de résistance vis-à-vis de ces molécules peut être variable d'un pays à un autre selon les différentes politiques de thérapie antimicrobienne et les antécédents aux antibiotiques des populations (Waites et al., 2003 ; Farkas et al., 2011 ; Karou et al., 2012). En Côte d'Ivoire, très peu d'études ont porté sur la résistance des souches de mycoplasmes. Dans un contexte global d'accroissement de la résistance aux antibiotiques, il était utile d'évaluer la sensibilité aux antibiotiques pour une meilleure prise en charge des infections à mycoplasmes. C'est dans cette perspective que s'inscrit cette étude qui avait pour objectif de déterminer le niveau de sensibilité aux antibiotiques des mycoplasmes identifiés au cours de spermocultures réalisées chez des patients adressés au laboratoire d'hygiène de l'Institut National d'Hygiène Publique (INHP) dans le cadre de l'exploration biologique des troubles de la fertilité.

\section{MATERIEL ET METHODES \\ Matériel}

Il s'agit d'une étude rétrospective portant sur les mycoplasmes isolés à partir des 
prélèvements de sperme de patients adressés au laboratoire d'hygiène de l'INHP de janvier 2015 à décembre 2016. Les prélèvements ont été réalisés au laboratoire et les analyses ont été effectuées selon les instructions du fabricant. L'identification des germes et l'évaluation de leur sensibilité aux antibiotiques ont été réalisées grâce au kit Mycoplasma $\mathrm{IST}_{2}$ (Biomérieux, Réf 42 505). Ce kit associe un bouillon de culture sélectif à une galerie comprenant 22 tests. Il permet la culture, l'identification, la numération et l'étude de la sensibilité de neuf antibiotiques appartenant à trois familles distinctes: les cyclines (doxycycline et tétracycline), les macrolides et apparentés (josamycine, érythromycine, clarithromycine, azithromycine et pristinamycine) ainsi que les fluoroquinolones (ofloxacine et ciprofloxacine).

\section{Méthodes}

L'identification des mycoplasmes est basée sur les propriétés métaboliques spécifiques de chacune des espèces (hydrolyse de l'urée pour $U$. urealyticum et hydrolyse de l'arginine pour M. hominis); la présence de ces bactéries est caractérisée par un changement de couleur de l'indicateur de $\mathrm{pH}$ (rouge de phénol) du jaune orangé au rouge ou au violet pour un taux de mycoplasmes supérieur à $10^{4}$ unités formant colonies par millilitre. En cas de co-infection, un virage de la couleur était noté dans les deux puits permettant l'identification de $U$. urealyticum et de M. hominis ;

L'étude de la sensibilité aux antibiotiques reposait sur la présence ou non de culture bactérienne dans les différents puits contenant les antibiotiques, se traduisant par un virage de l'indicateur de $\mathrm{pH}$ en cas de croissance.

\section{RESULTATS}

Prévalence des mycoplasmes urogénitaux

Durant la période d'étude, 858 échantillons de sperme ont été analysés dont 313 étaient positifs pour les mycoplasmes soit un taux de positivité de 36,5\%. Les agents d'infections sexuellement transmissibles (IST) associés à l'infection à mycoplasmes étaient par ordre de fréquence décroissant : Chlamydia trachomatis (20,8\%) et Gardenerella vaginalis (9,6\%). De façon globale, U. urealyticum et Mycoplasma hominis ont été respectivement retrouvés dans $286(91,4 \%)$ et $68(7,9 \%)$ échantillons. $U$. urealyticum a été identifié de façon isolé dans 245 cas $(78,3 \%)$ et $M$. hominis dans 27 $(8,6 \%)$ alors que la co-infection $U$. urealyticum/M. hominis a été retrouvée chez 41 sujets soit $13,1 \%$ (Tableau 1 ).

\section{Sensibilité aux antibiotiques}

Concernant la sensibilité aux antibiotiques (Tableau 2), la josamycine était la molécule la plus active quel que soit le germe considéré et même en co-infection avec des sensibilités respectives de 90,2\% et $92,6 \%$ pour $U$. urealyticum et $M$. hominis. Dans ce travail, $81,5 \%$ des souches de $M$. hominis étaient sensibles à l'azithromycine bien que cette espèce soit naturellement résistante (Waites et al., 2012). La pristinamycine a également montré une efficacité sur $70,4 \%$ des souches de $M$. hominis. Les cyclines ont gardé une bonne efficacité sur $U$. urealyticum avec $71,4 \%$ de souches sensibles à la tétracycline et $76,7 \%$ à la doxycycline; l'activité sur Mycoplasma hominis était plus faible ( $44 \%$ et $63 \%$ ). Les fluoroquinolones (ofloxacine, ciprofloxacine) avaient l'activité la plus faible vis-à-vis des souches de $U$. urealyticum $(15 \%)$; cette activité était moyenne sur les souches de $M$. hominis (59\% et $63 \%$ ). 
Tableau 1 : Prévalence des mycoplasmes urogénitaux identifiés.

\begin{tabular}{l|cc}
\hline Espèces identifiées & n & \% \\
\hline Ureaplasma urealyticum & 245 & $78,3 \%$ \\
Mycoplasma hominis & 27 & $8,6 \%$ \\
U. urealyticum et M. hominis & 41 & $13,1 \%$ \\
\hline Total & 313 & $100,0 \%$ \\
\hline
\end{tabular}

Tableau 2 : Sensibilité des souches de $U$. urealyticum et $M$. hominis aux antibiotiques.

\begin{tabular}{l|cccccc}
\hline & \multicolumn{2}{c}{$\mathbf{U U}(\mathbf{n}=\mathbf{2 4 5})$} & \multicolumn{2}{c}{$\mathbf{M H}(\mathbf{n = 2 7})$} & \multicolumn{2}{c}{$\mathbf{U} / \mathbf{M H}(\mathbf{n}=\mathbf{4 1})$} \\
& $\mathbf{N}$ & $\mathbf{\%}$ & $\mathbf{N}$ & $\mathbf{\%}$ & $\mathbf{N}$ & $\%$ \\
\hline Tétracycline & 175 & 71,4 & 12 & 44,4 & 22 & 53,7 \\
Doxycycline & 188 & 76,7 & 17 & 63 & 25 & 61 \\
Erythromycine & 162 & 66,1 & 6 & 22,2 & 14 & 34,1 \\
Josamycine & 221 & 90,2 & 25 & 92,6 & 34 & 82,9 \\
Azithromycine & 227 & 92,7 & 22 & 81,5 & 30 & 73,2 \\
Clarithromycine & 139 & 56,7 & 9 & 33,3 & 18 & 43,9 \\
Pristinamycine & 135 & 55,1 & 19 & 70,4 & 20 & 48,8 \\
Ofloxacine & 39 & 15,9 & 16 & 59,3 & 9 & 22 \\
Ciprofloxacine & 37 & 15,1 & 17 & 63 & 8 & 19,5 \\
\hline
\end{tabular}

\section{DISCUSSION}

Dans la présente étude, l'identification et l'évaluation de la sensibilité aux antibiotiques de $U$. urealyticum et de $M$. hominis ont été réalisées à partir de prélèvements de sperme. Le taux de positivité aux mycoplasmes était de 36,5\%. Ce taux est proche de celui trouvé par Gandji et al. (2013) qui était de $35,1 \%$ mais inférieur à celui trouvé par Xiaofei et al. (2016) qui était de $43,7 \%$. U. urealyticum était prédominant avec un taux d'identification de $91,4 \%$ contre $21,7 \%$ pour $M$. hominis. De même, la coinfection $U$. urealyticum / M. hominis était supérieure à la mono-infection par $M$. hominis. Ces résultats sont en accord avec ceux de nombreux auteurs, avec toujours une part plus importante d'infections à $U$. urealyticum (Karou et al., 2012 ; Gandji et al., 2013 ; Diaz et al., 2013 ; Diop-N'diaye et al., 2014 ; Xiaofei et al., 2016). Ces résultats sont contraires à celui de Ouégnin et al. (2008), qui ont obtenu une prédominance en faveur de M.hominis avec $39,9 \%$ contre $11,7 \%$ pour $U$. urealyticum.

Concernant la sensibilité aux antibiotiques, M. hominis et U. urealyticum sont connus pour présenter des sensibilités différentes aux antibiotiques avec une résistance naturelle de $M$. hominis aux macrolides en C14 (érythromycine, clarithromycine) et $\mathrm{C} 15$ (azithromycine) tandis que $U$. urealyticum est naturellement résistant aux lincosamides (lincomycine) (Waites et al., 2012). Cependant dans ce travail, $81,5 \%, 33,3 \%$ et $22,2 \%$ des souches 
de $\quad$ M. hominis étaient sensibles respectivement à l'azithromycine, à la clarithromycine et à l'érythromycine. D'autres auteurs ayant évalué la sensibilité aux antibiotiques des mycoplasmes à partir de tests de micro dilution en milieu liquide, ont rapporté des taux de sensibilité non négligeables vis-à-vis des macrolides en C14 et C15 (Karou et al., 2012 ; Zhu et al., 2012 ; Diaz et al., 2013). De même, l'utilisation de culture en milieu liquide à partir de prélèvements rend complexe l'interprétation de la résistance en cas de co-infection, car il est impossible de rendre compte de la sensibilité de chacune des espèces. Ceci souligne les limites de ces tests de sensibilité pour mycoplasmes et la nécessité de contrôler la qualité des tests (Waites et al., 2012). La josamycine était la molécule efficace contre les deux espèces même lorsqu'elles étaient en co-infection. Ce constat a été fait avec DiopN'diaye et al. (2014) et Xiaofei et al. (2016). En ce qui concerne les cyclines, des résistances acquises liées à la présence d'un transposon conjugatif contenant le gène tet (M) sont signalées depuis plus d'une vingtaine d'années; Il s'agirait de résistances croisées entre la tétracycline, la doxycycline et la minocycline et concernent $5 \%$ environ des souches de $U$. urealyticum et de $M$. hominis (Diop-N'diaye et al. (2014). Dans ce travail, le niveau de résistance aux cyclines était plus élevé avec $M$. hominis et était respectivement de $37 \%$ et $55 \%$ pour la tétracycline et la doxycycline ; pour $U$. urealyticum, il était de $29 \%$ pour la tétracycline et de $23 \%$ pour la doxycycline. Ce niveau de résistance est supérieur à ceux obtenus à Dakar par SOW et al. (2000) et Diop-N'diaye et al. (2014) avec des taux qui variaient entre $5 \%$ et $14 \%$. Nos résultats se rapprochent de ceux obtenus au Burkina Faso par Karou et al. (2012) et à Cuba par Diaz et al. (2013) avec des taux de résistance à la tétracycline et à la doxycycline respectivement de $20 \%$ et $25 \%$ pour $U$. urealyticum et $40 \%$ et $35 \%$ pour M. hominis. A l'inverse, dans l'étude de Xiaofei et al. (2016) en Chine, les cyclines ont été les molécules qui avaient montré la meilleure activité sur l'ensemble des mycoplasmes isolés, aussi bien en mono-infection qu'en coinfection. Cette augmentation de la résistance pourrait s'expliquer par la pression de sélection exercée par les cyclines qui restent les médicaments de première ligne dans le cadre de l'approche syndromique du traitement des IST en Côte d'Ivoire. Par ailleurs, le taux de sensibilité d'Ureaplasma urealyticum vis-à-vis de l'érythromycine (66\%) est inférieur aux résultats de Sow et al. (2000) et Diop-N'diaye et al. (2014) à Dakar qui ont rapporté des niveaux de sensibilités supérieures à 93\%. Dans notre série, les fluoroquinolones ont présenté une faible activité vis-à-vis des souches d'U. urealyticum tandis que cette activité était relativement moyenne avec $M$. hominis. Ces résultats se rapprochent de ceux de Xiaofei et al. (2016) en Chine et opposés à ceux de Diop-N'diaye et al. (2014) à Dakar qui rapporte une efficacité supérieure à $94 \%$ sur les deux espèces de mycoplasmes. La résistance aux quinolones résulte essentiellement de la sélection de mutations au niveau de l'ADN gyrase ou de la topoisomérase IV consécutive à l'utilisation à grande échelle de ces molécules. Ainsi, du fait d'une très large prescription de ces antibiotiques, la sensibilité aux fluoroquinolones est largement en baisse dans de nombreux pays avec des taux de résistance pouvant atteindre dans certaines zones $70 \%$ (Zhu et al., 2012; Diaz et al., 2013; De Francesco et al., 2013). Toutefois, ces différences de prévalence de souches résistantes ne sont que le reflet des différentes politiques de prises en charge des infections mises en place dans les pays sans oublier les pratiques d'automédication très répandues dans les pays africains qui conditionnent les pressions de sélection sur les populations microbiennes (Hounsa et al. , 2010 ; Hailaji et al. , 2016).

\section{Conclusion}

La présente étude qui avait pour objectif d'évaluer le niveau de sensibilité aux antibiotiques des mycoplasmes identifiés au cours de la spermoculture réalisée chez des patients présentant des troubles de la fertilité, 
a permis de montrer que les cyclines conservaient encore une bonne efficacité visà-vis des souches de $U$. ureaplasma malgré une baisse de sensibilité liée à leur large utilisation dans le cadre de l'approche syndromique du traitement des IST. La josamycine est restée active sur les deux espèces de mycoplasmes, en mono ou coinfections. Le niveau de résistance des souches de $U$. urealyticum vis-à-vis des fluoroquinolones était très élevé (85\%). Pour une meilleure prise en charge des infections à mycoplasmes, il faut nécessairement faire une bonne identification des espèces du fait de leur différence de sensibilités aux antibiotiques et une bonne standardisation des méthodes compte tenu des divergences qui ont été observées d'une étude à une autre.

\section{CONFLIT D'INTERETS}

Les auteurs déclarent ne pas avoir de conflit d'intérêts en relation avec cet article.

\section{CONTRIBUTIONS DES AUTEURS}

TBKA a contribué à la réalisation des analyses, à l'exploitation des résultats et à la rédaction du manuscrit. AMC, AGK et NYZ ont contribué à la correction du manuscrit. CNA a corrigé et supervisé les travaux.

\section{REMERCIEMENTS}

Le Laboratoire remercie les techniciens N'difon Kopoin et Yedmel Augustin pour leur contribution à l'analyse des échantillons.

\section{REFERENCES}

Abarikwu SO. 2013.Causes and Risk Factors for Male-Factor Infertility in Nigeria: A Review. African Journal of Reproductive Health, 17(4): 150-166.

Abdel Rahman MS, Hassan RA, Sakna NA. 2016. Antimicrobial susceptibility pattern of genital. Mycoplasmas among a group of pregnant women. Alexandria Journal of Medicine, 52: 353-358. http://dx.doi.org/10.1016/j.ajme.2015.12. 005

De Francesco MA, Caracciolo S, Bonfanti C, Manca N. 2013. Incidence and antibiotic susceptibilty of Mycoplasma hominis and Ureaplasma urealyticum isolated in Brescia, Italy, over 7 years. $J$ Infect Chemother., 19(4): 621-627. DOI: 10.1007/s10156-012-0527-z

Diaz L, Cabrera LE, Fernandez T, Ibanez I, Torres Y, Obregon Y, Rivero Y. 2013. Frequency and antimicrobial sensitivity of Ureaplasma urealyticum and Mycoplasma hom201inis in patients with vaginal discharge. MEDICC Rev., 15(4): 45-47.

Diop-N'diaye H, Gueye M, Camara M, Gueye SB, Ba-Diallo A, Karam F, DiagneSamb H, Fall-Niang MY, Diouf O, Toure-Kane NC, Mboup S, Gaye-Diallo A. 2014. Sensibilité aux antibiotiques de Mycoplasmahominis et deUreaplasma urealyticum isolées du tractus génital de femmes à Dakar. Dakar Med., 59(3): 177-184.

Farkas B, Ostorhazi E, Ponyai K, Toth B, Adlan E, Parducz L, Marschalko M, Karpati S, Rozgonyi F. 2011. Frequency and antibiotic resistance of Ureaplasma urealyticum and Mycoplasma hominis in genital samples of sexually active individuals. Orv Hetil., 152(42): 16981702. DOI: $10.1556 / \mathrm{OH} .2011 .29217$

Gandji S, Bankole HS, Dougnon TV, Da Silva J, Zannou C, Biaou O. 2013. Survenue des obstructions tubaires chez les femmes à Cotonou (Bénin) : rôle des bactéries. Int. J. Biol. Chem. Sci., 7(3): 1338-1343.

DOI: http://dx.doi.org/10.4314/ijbcs.v7i3.39

Hailaji NSM, Ould Salem ML, Ghaber SM. 2016. La sensibilité aux antibiotiques des bactéries uropathogènes dans la ville de Nouakchott-Mauritanie. Prog Urol. 26(6): 346-352. DOI: https://doi.org/10.1016/j.purol.2016.04.0 04

Hartmann M. 2009. Genital mycoplasmas. $J$ Dtsch Dermatol Ges., 7(4): 371-377. DOI: $\quad 10.1111 / \mathrm{j} .1610-$ 0387.2008.06965.x.

Hounsa A, Kouadio L, De Mol P. 2010. Automédication par les antibiotiques provenant des pharmacies privées de la ville d'Abidjan en Côte d'Ivoire. Med 
Mal Infect., 4(6): 333-340. Doi : 10.1016/j.medmal.2009.10.002.

Karou SD, Djigma F, Sagna T, Nadembega C, Zeba M, Kabre A, Anani K, Ouermi D, Gnoula C, Pietra V, Pigatelli S, Simpore J. 2012. Antimicrobial resistance of abnormal vaginal discharges microorganisms in Ouagadougou, Burkina Faso. Asian Pac J Trop Biomed., 2(4): 294-297.

Nana PN,Wandji JC, Fomulu JN, Mbu RE, Leke RJI, Woubinwou MJ. 2011. Aspects Psycho-Sociaux chez Patients Infertiles à la Maternite Principalede l'Hopital Central de Yaoundé, Cameroun. Clinics in Mother and Child Health, 8: $1-5 . \quad$ DOI: 10.4303/cmch/C100601

Nwadioda S, Egesie JO, Emejuo H, Iheanacho E. 2010. A study of female genital swabs in Nigerian tertiary hospital. Asian Pac J Trop Med., 3(7): 577-579. https://doi.org/10.1016/S19957645(10)60140-X

Ouegnin GA, Gnagne YM, Vodi CC, FayeKette H, N'douba KA, Tchetche RG. 2008. Rôle des germes de l'infection génitale dans l'infertilité masculine. Revue Bio-Africa., 6: 32-38.

Sow AI, Diallo Y, Diab El Hadi A, Samb A. 2000. Sensibilité in vitro aux antibiotiques de 178 souches de mycoplasmes génitaux isolées chez des consultantes en gynécologie à Dakar. Bull Soc Pathol Exot., 93(1): 6-7.

Waites KB, Crabb DM, Bing X, Duffy LB. 2003. In vitro susceptibilities to and bactericidal activities of garenoxacin (BMS-284756) and other antimicrobial agents against human Mycoplasmas and Ureaplasmas. Antimicrob Agents Chemother., 47(1): 161-165. DOI: 10.1128/AAC.47.1.161-165.2003.

Waites KB, Duffy LB, Bebear CM, Matlow A, Talkington DF, Kenny GE, Totten PA, Bade DJ, Zheng X, Davidson MK, Shortridge VD, Watts JL, Brown SD. 2012. Standardized methods and quality control limits for agar and broth microdilution susceptibility testing of Mycoplasmapneumoniea, Mycoplasma hominis and Ureaplasma urealyticum. $J$ Clin Microbiol., 50(11): 3542-3547. DOI: $10.1128 / \mathrm{JCM} .01439-12$

Xiaofei Z, Min L, Huiling C, Xuewen Y, Chunbing Z. 2016. Epidemiology of Ureaplasma urealyticum and Mycoplasma hominis in the semen of male outpatientswith reproductive disorders. Exp Ther Med., 12(2): 11651170. DOI: 10.3892/etm.2016.3409.

Zhu C, Liu J, Ling Y, Dong C, Wu T, Yu X, Hou Y, Dong L, Cheng X. 2012. Prevalence and antimicrobial susceptibility of Ureaplasma urealyticum and Mycoplasma hominis in Chinese women with genital infectious diseases. Indean J Dermatol Venereol Leprol., $\quad$ 78(3) : 406-407. DOI: 10.4103/0378-6323.95480. 